\title{
Evaluation of Periodontal Space Widening in Patients with Antiresorptive Drug-related Osteonecrosis of the Jaws (ARONJ) on Panoramic Radiographs
}

\author{
ALEXANDRE T. ASSAF*, VIVIEN AMBERG*, RALF SMEETS, JOHANNES WIKNER, HENNING HANKEN, \\ JAN SEMMUSCH, FLORIAN EWALD, ASHKAN RASHAD and REINHARD E. FRIEDRICH \\ Department of Oral and Maxillofacial Surgery, University Medical Center Hamburg Eppendorf, \\ University of Hamburg, Hamburg, Germany
}

\begin{abstract}
Background/Aim: The purpose of this study was to evaluate a potential widening of the periodontal space as an initial measurable imaging criterium on panoramic radiographs in patients with the diagnosis of antiresorptive drug related osteonecrosis of the jaw (ARONJ). Patients and Methods: A retrospective analysis of panoramic radiographs of 16 patients (12 females and 4 males; mean age is 70.5 years, standard deviation is 14 years) was performed, over a period of 12 months with the diagnosis of ARONJ. Panoramic radiographs of 16 healthy patients (12 females and 4 males; mean age was 70.6 years, standard deviation was 13.8 years) without diagnosis of ARONJ served as controls. All images were taken with the same device and were evaluated by two experienced maxillofacial surgeons and one dentist. Results: Compared to the control group, a mean widening of the periodontal space of $0.06 \mathrm{~mm}$ (confidence interval $(\mathrm{CI})=0.05-0.17 \mathrm{~mm})$ was found in the study group. However, this difference was not statistically significant. Conclusion: Although a very extensive and sophisticated interindividual comparison was performed to evaluate for slight changes of the periodontal space in patients with antiresorptive drug therapy, our results demonstrate that PDS widening is not a predictive parameter for ARONJ. Therefore, our results indicate that panoramic
\end{abstract}

*These Authors contributed equally to this study.

Correspondence to: Alexandre Thomas Assaf, MD, DMD, Ph.D., FEBOMFS, Department of Oral and Maxillofacial Surgery, University Medical Center Hamburg Eppendorf, Martinistrasse 52, 20246 Hamburg, Germany. Tel: +49 40741053259, Fax: +49 40741055467,e-mail: a.assaf@uke.de

Key Words: Antiresorptive drug therapy, antiresorptive drug related osteonecrosis of the jaw (ARONJ), bisphosphonate (BP), denosumab (Dmab), panoramic radiographs (PR), periodontal space widening (PSW), periodontal space (PDS). radiographs are not sufficient enough to allow assessment of stages and disease progress in ARONJ patients regarding to periodontal space widening.

The diagnosis of bisphosphonate related osteonecrosis of the jaws (BRONJ) was firstly described in 2003 by Robert E. Marx (1). The term of this still increasing number of patients with an osteonecrosis was adapted to antiresorptive drug related osteonecrosis of the jaws (ARONJ), since additional drugs (e.g. RANKL-inhibitors such as Denosumab (Dmab)) were introduced for the clinical treatment of bone diseases, such as osteoporosis, osteitis deformans (Paget's disease) and malignant diseases, e.g. multiple myeloma or osseous metastasis $(2,3)$.

However, several studies $(1,4,5)$ demonstrated that especially the intravenous application of these medications was followed by ARONJ. Denosumab blocks the receptormediated activation of osteoclasts and has no affinity to the bone matrix (5). On the contrary bisphosphonates (BPs) bind to calcium (6) and hydroxyapatite (7), which are the main contents of the bone. Both types of antiresorptive drugs (BPgroup and Dmab) correlate with the induction of ARONJ (8). Furthermore, certain BPs such as zoledronate seem to be more effective than others $(6,9)$, but also seem to have a higher risk for the occurrence of ARONJ, which was demonstrated in one of our recent studies (10). Zoledronate was also most frequently used on patients with ARONJ in this study.

The frequency of occurrence of ARONJ described in the literature ranges from $1 \%$ to $20 \%$ after intravenous application in patients with multiple myeloma or bone metastasis (11).

The incidence of ONJ in oncology patients treated i.v. with BPs ranges from 0 to 12,222 per 100,000 patient-years (12-16), and the incidence of ONJ in oncology patients treated with Dmab ranges from 0 to 2,316 per 100,000 patient-years $(15,17-23)$. 
ARONJ is most commonly diagnosed by detailed intraoral and extraoral examination by the dentist or the oral and maxillofacial surgeon combined with radiographic examination using panoramic radiographs, combined with 3D-examinations such as computed tomography scans (CT), cone beam computed tomography (CBCT) or magnetic resonance imaging (MRI) (24, 25). Supplementary investigations might be helpful to better evaluate the extension of the necrotic area, by using scintigraphy or single photon emission computed tomography (SPECT) (10).

Typical symptoms in ARONJ patients are non-healing wounds, e.g. after intraoral procedures like tooth extractions, but also spontaneously in close proximity to healthy teeth (4). Osteonecrotic lesions can also be seen in patients with partial or complete dentures, caused by punctual or selective distress to the gingiva or maxillo-mandibular bone $(26,27)$. Those osteonecrotic lesions occur most common in the posterior portion of the mandible (26) in which very thin mucosa separates the jaw bone from the oral cavity $(1,28)$.

The American Association of Oral and Maxillofacial Surgeons (AAOMS) has conducted a summary of ARONJ criteria in the recent updated version of the initial positioning paper from 2014. Herein, a former or current treatment with BPs or Dmab, no radiotherapy and metastasis in the head and neck area, and an occurrence of exposed bone for more than eight weeks is required for the classification of ARONJ (5).

Compared to the initial versions from 2007 and 2009 in which criteria of diagnosis included patients with indefinite odontalgia, bone pain, loosening of teeth, periodontal fistula without any inflammation signs, or disorders which are attributed to dental causes, the latest version from 2014 also included patients at risk with no evidence of exposed bone but former treatment of antiresorptive drug therapy (5).

As described by several authors, one of the major initial risk factors is dental or oral surgery with direct contact to the jaw bone $(1,25,28)$. Even after surgical debridement of osteonecrotic lesions, reoccurrence of exposed bone frequently occurs with disseminated infections, sometimes resulting in the necessity of partial or complete resection of the mandible and combined microsurgical reconstruction $(4,28)$. Recently, cytostatic therapy was described as a potential risk factor for the occurrence of ARONJ. Several authors described a correlation between the use of drugs such as Dmab or bevacizumab and the occurrence of ARONJ $(29,30)$.

Regarding the selected studies, saliences such as thickening of the lamina dura and the alveolar crest or sclerosis of the alveolar margin with a constriction of the mandibular nerve canal, can be detected by radiographic examination (31). Fleisher et al. described a correlation between the widening of the periodontal ligament and the occurrence of ARONJ, which was found in approximately $80 \%$ of patients (32).

The aim of this study was to verify or disprove this idea of PSW by measuring and analyzing it on panoramic radiographs in patients with ARONJ at stages I or II and comparing it to patients at the same age and gender without antiresorptive drug therapy.

\section{Patients and Methods}

During a period of 12 months (June 2014 till June 2015) a total of 16 patients out of 57 with the diagnosis of ARONJ were included in this retrospective evaluation (12 females (75\%) and 4 males $(25 \%))$. The study was conducted at the Department of Oral and Maxillofacial Surgery at the University Medical Center Hamburg Eppendorf. The data included retrospective evaluation of patients' medical history and panoramic radiographs.

Inclusion criteria for the ARONJ-group (Group A) were patients with ARONJ diagnosis at stages I or II with shortened dentition and symptoms such as exposed bone, non- healing extraction wounds or sequestration of the bone, which cannot be associated with odontogenic infections. For the non-ARONJ-group (Group B), patients were selected to be at same age and gender like the patients from Group A, with exactly the same teeth existing, as measured in Group A (Table I).

Patients in group A have been referred to our clinic with the initial diagnosis of a BP- or Dmab- induced osteonecrosis of the jaws (ARONJ).

The control group consisted of 16 patients (12 females (75\%) and 4 males $(25 \%)$ ), being at the same age but without any history of antiresorptive drug therapy, radiation therapy or evidence of ARONJ. All patients of this group received their panoramic radiographs due to other causes, but without any signs of oral diseases.

Furthermore, the medical history of each patient was evaluated by including the date of initial diagnosis with ARONJ, type of disease, comorbidities, metastases, exclusion of radiation therapy in the head and neck region, or any other use of cytostatics, antibiotics or immunosuppressive therapy. Therefore, a total of 41 patients out of $57(71.9 \%)$ were excluded from this retrospective evaluation.

Radiographic analysis and measuring of the periodontal space. All radiographic images were taken with the same device at the same department of oral and maxillofacial surgery (VeraView IC5 HD, J. Morita MFG CORP., 2010, exposure parameters: - 68 kV/6 mA approximately value for adults; variable through automatic exposure control; dose area product: $60 \mathrm{mGy} / \mathrm{cm}^{2}$ ). All panoramic radiographs were analyzed regarding the radiographic appearance of periodontal space widening (PSW). The measurements of the periodontal ligament were taken on each tooth: six measurements from the alveolar bone towards the root. The root of the tooth was divided into three parts, the first (coronal part), second (medial part) and third (apical part). The measurements were then taken mesial and distal as far as legible.

Also, oral conditions of each patient were analyzed and evaluated. Radiographic abnormalities were documented, particularly the evidence of osteonecrosis/osteosclerosis, persisting alveolar sockets with unremodeled bone, such as fillings, tooth canal treatments, crowns, bridges, implants, caries, inflammatory periapical lesions and other saliences.

Data evaluation. The panoramic views were imported to Osirix imaging software, (Version 5.6, Pixmeo SARL, CH-1233 Bernex, Switzerland), which is an image processing software dedicated to 
Table I. Overview and medical history of the 16 patients participated in this study with ARONJ.

\begin{tabular}{|c|c|c|c|c|c|c|c|c|c|c|}
\hline $\begin{array}{l}\text { Patient } \\
\text { No. }\end{array}$ & Age & Gender & $\begin{array}{l}\text { Initial } \\
\text { diagnosis }\end{array}$ & Metastases & Comorbidities & $\begin{array}{c}\text { Bisphosphonate } \\
\text { or denosumab }\end{array}$ & $\begin{array}{l}\text { Cytostatic } \\
\text { therapy }\end{array}$ & $\begin{array}{c}\text { Distant } \\
\text { radiation }\end{array}$ & $\begin{array}{c}\text { Immunosuppressiv } \\
\text { therapy }\end{array}$ & ve Antibiotics \\
\hline 1 & 85 & $\mathrm{f}$ & $\begin{array}{l}\text { Multiple } \\
\text { myeloma }\end{array}$ & Yes & $\begin{array}{l}\text { Hyperthyreosis, } \\
\text { hypercholesterolemia, } \\
\text { arterial hypertension }\end{array}$ & Zoledronate & Bortezomib & No & Prednisolone & Amp/Sulb \\
\hline 2 & 78 & $\mathrm{f}$ & Osteoporosis & - & $\begin{array}{l}\text { Osteoporosis, } \\
\text { rheumatism }\end{array}$ & Denosumab & - & No & - & Amp/Sulb \\
\hline 3 & 86 & $\mathrm{f}$ & Breast cancer & Yes & $\begin{array}{c}\text { Arterial } \\
\text { hypertension }\end{array}$ & Zoledronate & $\begin{array}{l}\text { Paclitaxel + } \\
\text { epirubicin }\end{array}$ & No & - & Clindamycin \\
\hline 4 & 78 & $\mathrm{~m}$ & $\begin{array}{l}\text { Colorectal and } \\
\text { prostate cancer }\end{array}$ & Yes & $\begin{array}{c}\text { Arterial } \\
\text { hypertension }\end{array}$ & $\begin{array}{c}\text { Zoledronate/ } \\
\text { denosumab }\end{array}$ & Trenantone & No & - & $\begin{array}{l}\text { Amp/Sulb + } \\
\text { Doxycycline }\end{array}$ \\
\hline 5 & 68 & $\mathrm{f}^{\mathrm{t}}$ & Breast cancer & Yes & $\begin{array}{l}\text { Giant cell } \\
\text { arteritis }\end{array}$ & Zoledronate & Anastrozol & No & $\begin{array}{c}\text { Prednisolone }+ \\
\text { MTX }\end{array}$ & Clindamycin \\
\hline 6 & 75 & $\mathrm{f}$ & Osteoporosis & - & $\begin{array}{c}\text { Diabetes, } \\
\text { hypertension, } \\
\text { arthritis pancreatic } \\
\text { insufficiency }\end{array}$ & Alendronate & - & - & - & Amp/Sulb \\
\hline 7 & 80 & $\mathrm{f}$ & Breast cancer & Yes & - & Zoledronate & - & Yes & - & Amp/Sulb \\
\hline 8 & 71 & $\mathrm{f}$ & Breast cancer & Yes & $\begin{array}{c}\text { Hyperthyreosis, } \\
\text { lymphocytic } \\
\text { alveolitis }\end{array}$ & Zoledronate & No & Yes & Prednisolone & Amp/Sulb \\
\hline 9 & 78 & $\mathrm{f}$ & Breast cancer & Yes & $\begin{array}{c}\text { Diabetes, } \\
\text { arterial hypertension, } \\
\text { pulmonary embolism, } \\
\text { arthritis, } \\
\text { spinal stenosis, } \\
\text { chronic hepatitis }\end{array}$ & Zoledronate & Tamoxifen & Yes & Prednisolone & - \\
\hline 10 & 62 & $\mathrm{f}$ & Osteoporosis & - & $\begin{array}{l}\text { Cardiac arrhythmia, } \\
\text { heart valve disease }\end{array}$ & Alendronate & no & - & - & $\begin{array}{c}\text { Clindamycin }+ \\
\text { Moxifloxacin }+ \\
\text { Amp/Sulb }\end{array}$ \\
\hline 11 & 54 & $\mathrm{f}$ & Breast cancer & Yes & - & Ibandronate & $\begin{array}{c}\text { FEC + } \\
\text { Tamoxifen + } \\
\text { Anastrozol }\end{array}$ & $\begin{array}{c}\text { No } \\
\text { (Denied) }\end{array}$ & - & Clindamycin \\
\hline 12 & 75 & $\mathrm{~m}$ & $\begin{array}{l}\text { Prostate } \\
\text { cancer }\end{array}$ & Yes & $\begin{array}{l}\text { Bronchial asthma, } \\
\text { arterial hypertension, } \\
\text { auricular fibrillation }\end{array}$ & Alendronate & - & - & - & Penicillin \\
\hline 13 & 85 & $\mathrm{f}$ & Renal cancer & Yes & Hyperthyreosis & Ibandronate & Sunitinib & Yes & - & $\begin{array}{l}\text { Amp/Sulb + } \\
\text { Doxycycline }\end{array}$ \\
\hline 14 & 62 & $\mathrm{~m}$ & Lung cancer & Yes & Oral cancer & Zoledronate & no & Yes & - & Amp/Sulb \\
\hline 15 & 32 & $\mathrm{f}$ & Osteomyelitis & - & Esophagitis & Alendronate & no & & - & $\begin{array}{c}\text { Cefuroxime+ } \\
\text { Amp/Sulb }\end{array}$ \\
\hline 16 & 66 & $\mathrm{~m} \mathrm{~F}$ & Prostate cancer & Yes & - & Zoledronate & no & Yes & Prednisolone & Amp/Sulb \\
\hline
\end{tabular}

FEC: 5-Fluorouracil, epirubicin and cyclophosphamide; Amp/Sulb: ampicillin + sulbactam; MTX: methotrexate.

DICOM images. It is specifically designed for navigation and visualization of multimodality and multidimensional images. All radiographs were first enlarged, then brightened and darkened for the best outcome.

Only images which allowed for at least three measurable data sets of one tooth were used. In the case of different interpretations, the images were reevaluated until consensus was attained.

\section{Results}

This retrospective study included 32 radiographic images of 32 different patients. The ARONJ-group (Group A) consisted of 16 patients, 12 females (75\%) and 4 males (25\%), who were treated with antiresorptive drugs and had a diagnosis of ARONJ according to the 2014 diagnosis criteria. The control group (Group B) also consisted of 16 patients (12 females and 4 males) with no history of antiresorptive drug medication, as well as no signs of any necrotic bone lesions due to previous radiation therapy. The mean age in both groups at time of examination was 70.6 years (range $=32-86$ years).

In group A, seven females were diagnosed with metastases from breast cancer $(43.75 \%)$, three with heavy and advanced osteoporosis (18.75\%), one with multiple myeloma $(6.25 \%)$ 


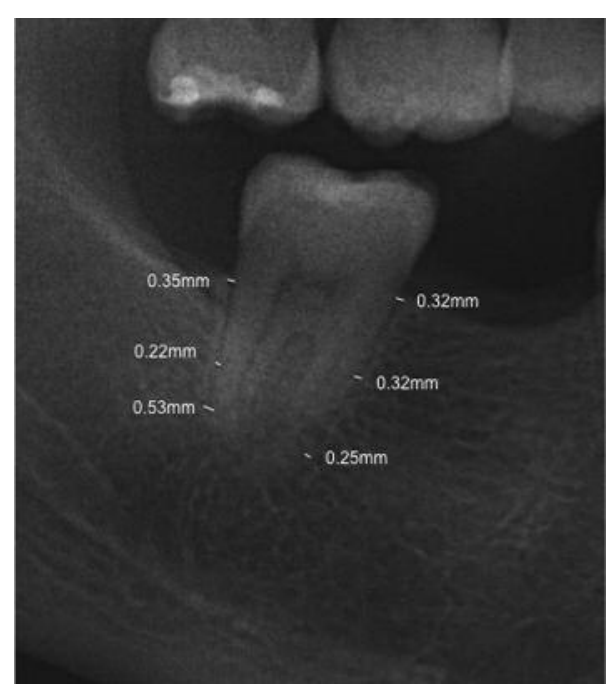

Figure 1. Enlarged section of a panoramic radiograph showing a part of the right side mandible of a 54-year-old female patient with ARONJ stadium II in region 36-37. Measuring of the periodontal space on tooth 47 (in mm: mesial from coronal to apical 0.32, 0.32, 0.25, and distal from coronal to apical $0.35,0.22,0.53)$ using OsiriX software.

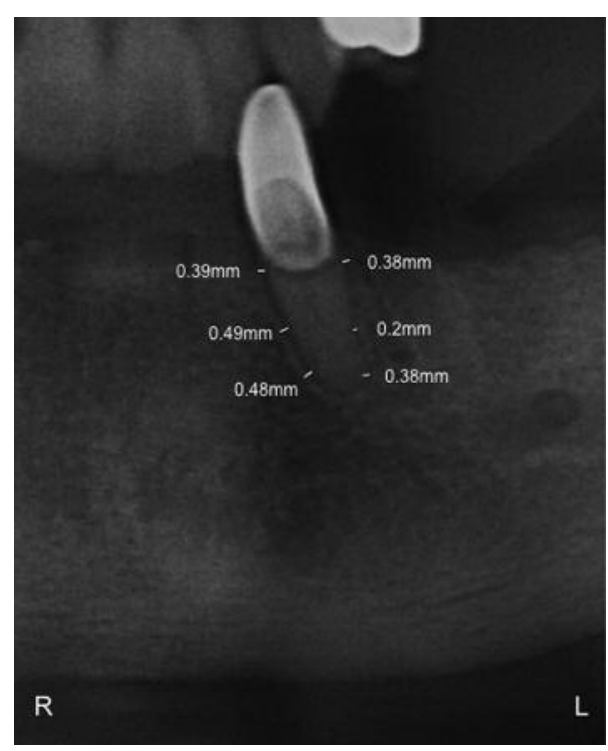

Figure 2. Enlarged section of a panoramic radiograph showing a part of the left side mandible of a 78-year-old male patient with ARONJ stadium II in region 36-37 after metastatic colorectal and prostate cancer after zoledronate and denosumab-therapy. Measuring of the periodontal space on tooth 33 (in mm: mesial from coronal to apical 0.39, 0.49, 0.48, and distal from coronal to apical $0.38,0.20,0.38$ ) using OsiriX software.

and one with osseous metastases from renal cancer $(6.25 \%)$. Among the male patients, two were diagnosed with metastases from prostate cancer (12.5\%), one had combined prostate cancer and a colorectal carcinoma (6.25\%) and one

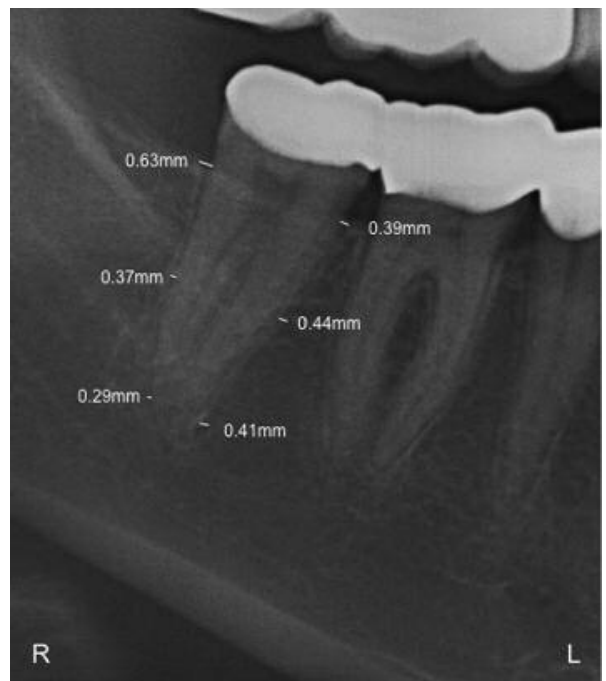

Figure 3. In comparison to figure 1 this Figure shows an enlarged section of a panoramic radiograph of the right-side mandible of a 54-year-old female patient without ARONJ. Measuring of the periodontal space on tooth 47 (in mm: mesial from coronal to apical 0.39, 0.44, 0.41, and distal from coronal to apical $0.63,0.37,0.29)$ using OsiriX software.

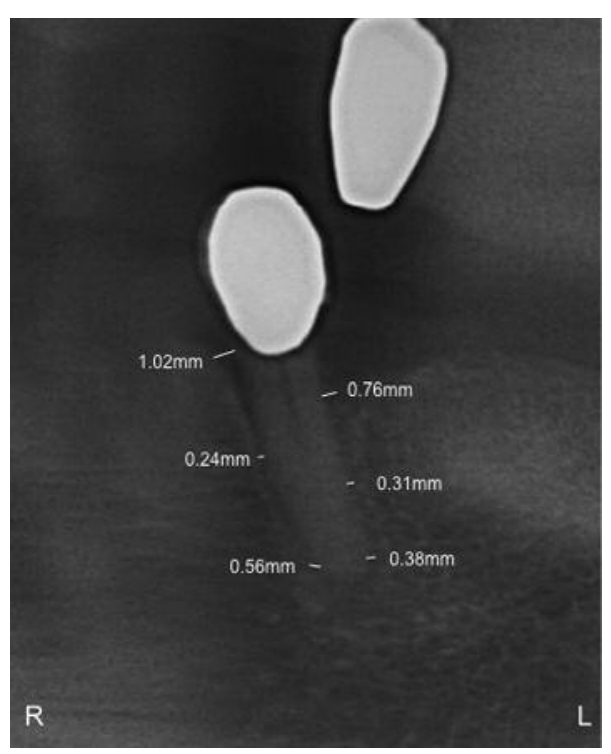

Figure 4. In comparison to Figure 3 this figure shows an enlarged section of a panoramic radiograph of the left side mandible of a 78year-old male patient without ARONJ. Measuring of the periodontal space on tooth 33 (in mm: mesial from coronal to apical 1.02, 0.24, 0.56 , and distal from coronal to apical 0.76, 0.31, 0.38) using OsiriX software.

suffered from lung cancer (6.25\%). A total of twelve patients had osseous metastasis $(75 \%)$ (Table I).

A total of 12 patients (75\%) from Group A received intravenous bisphosphonates or RANKL-inhibitors, 4 
Table II. Data of 16 patients with ARONJ (Group A).

\begin{tabular}{|c|c|c|c|c|c|c|c|c|}
\hline Patient No. & Number of teeth & Measured tooth & A & B & $\mathrm{C}$ & $\mathrm{D}$ & $\mathrm{E}$ & $\mathrm{F}$ \\
\hline 1 & 3 & 34 & $0.60 \mathrm{~mm}$ & $0.30 \mathrm{~mm}$ & $0.40 \mathrm{~mm}$ & & & \\
\hline 1 & 3 & 43 & $0.56 \mathrm{~mm}$ & $1.00 \mathrm{~mm}$ & $0.55 \mathrm{~mm}$ & & & $0.40 \mathrm{~mm}$ \\
\hline 1 & 3 & 44 & $0.40 \mathrm{~mm}$ & $0.60 \mathrm{~mm}$ & $0.50 \mathrm{~mm}$ & $0.30 \mathrm{~mm}$ & $0.50 \mathrm{~mm}$ & $0.40 \mathrm{~mm}$ \\
\hline 2 & 7 & 17 & $0.37 \mathrm{~mm}$ & & & & & \\
\hline 2 & 7 & 23 & $0.21 \mathrm{~mm}$ & & $0.24 \mathrm{~mm}$ & & $0.31 \mathrm{~mm}$ & \\
\hline 3 & 7 & 11 & & $0.87 \mathrm{~mm}$ & $0.36 \mathrm{~mm}$ & $0.31 \mathrm{~mm}$ & & $0.42 \mathrm{~mm}$ \\
\hline 3 & 7 & 21 & & $0.54 \mathrm{~mm}$ & & & & \\
\hline 3 & 7 & 22 & $0.31 \mathrm{~mm}$ & & $0.31 \mathrm{~mm}$ & & $2.00 \mathrm{~mm}$ & \\
\hline 3 & 7 & 23 & $0.68 \mathrm{~mm}$ & $0.51 \mathrm{~mm}$ & & & $0.21 \mathrm{~mm}$ & $0.30 \mathrm{~mm}$ \\
\hline 3 & 7 & 41 & $0.67 \mathrm{~mm}$ & $0.61 \mathrm{~mm}$ & $0.37 \mathrm{~mm}$ & $0.62 \mathrm{~mm}$ & $0.68 \mathrm{~mm}$ & $0.64 \mathrm{~mm}$ \\
\hline 3 & 7 & 42 & & $1.06 \mathrm{~mm}$ & & $0.73 \mathrm{~mm}$ & & \\
\hline 3 & 7 & 43 & $0.53 \mathrm{~mm}$ & $0.18 \mathrm{~mm}$ & & $0.19 \mathrm{~mm}$ & & \\
\hline 4 & 11 & 15 & $0.55 \mathrm{~mm}$ & & $0.11 \mathrm{~mm}$ & & $0.16 \mathrm{~mm}$ & \\
\hline 4 & 11 & 13 & $0.90 \mathrm{~mm}$ & $0.88 \mathrm{~mm}$ & $0.81 \mathrm{~mm}$ & $0.64 \mathrm{~mm}$ & & \\
\hline 4 & 11 & 33 & $0.39 \mathrm{~mm}$ & $0.63 \mathrm{~mm}$ & $0.44 \mathrm{~mm}$ & $0.37 \mathrm{~mm}$ & $0.41 \mathrm{~mm}$ & $0.29 \mathrm{~mm}$ \\
\hline 4 & 11 & 43 & $0.69 \mathrm{~mm}$ & $0.51 \mathrm{~mm}$ & $0.27 \mathrm{~mm}$ & $0.24 \mathrm{~mm}$ & $0.23 \mathrm{~mm}$ & $0.26 \mathrm{~mm}$ \\
\hline 5 & 2 & 43 & $1.30 \mathrm{~mm}$ & $7.40 \mathrm{~mm}$ & $1.80 \mathrm{~mm}$ & & $1.00 \mathrm{~mm}$ & \\
\hline 6 & 1 & 23 & $1.20 \mathrm{~mm}$ & $1.00 \mathrm{~mm}$ & $0.24 \mathrm{~mm}$ & $0.32 \mathrm{~mm}$ & $0.18 \mathrm{~mm}$ & \\
\hline 7 & 5 & 33 & $0.28 \mathrm{~mm}$ & $0.70 \mathrm{~mm}$ & $0.40 \mathrm{~mm}$ & $0.24 \mathrm{~mm}$ & $0.28 \mathrm{~mm}$ & $0.26 \mathrm{~mm}$ \\
\hline 7 & 5 & 32 & $0.27 \mathrm{~mm}$ & $0.70 \mathrm{~mm}$ & $0.46 \mathrm{~mm}$ & $0.37 \mathrm{~mm}$ & & $0.14 \mathrm{~mm}$ \\
\hline 7 & 5 & 41 & $0.24 \mathrm{~mm}$ & $0.46 \mathrm{~mm}$ & $0.35 \mathrm{~mm}$ & $0.29 \mathrm{~mm}$ & $0.26 \mathrm{~mm}$ & $0.18 \mathrm{~mm}$ \\
\hline 7 & 5 & 42 & $0.37 \mathrm{~mm}$ & $0.25 \mathrm{~mm}$ & $0.15 \mathrm{~mm}$ & $0.27 \mathrm{~mm}$ & & $0.27 \mathrm{~mm}$ \\
\hline 8 & 5 & 33 & $0.54 \mathrm{~mm}$ & & $0.28 \mathrm{~mm}$ & & $0.31 \mathrm{~mm}$ & \\
\hline 9 & 11 & 13 & $0.45 \mathrm{~mm}$ & $0.23 \mathrm{~mm}$ & & $0.51 \mathrm{~mm}$ & & \\
\hline 9 & 11 & 12 & $0.57 \mathrm{~mm}$ & $0.48 \mathrm{~mm}$ & & $0.39 \mathrm{~mm}$ & & \\
\hline 9 & 11 & 22 & $0.39 \mathrm{~mm}$ & $0.40 \mathrm{~mm}$ & $0.22 \mathrm{~mm}$ & $0.18 \mathrm{~mm}$ & $0.26 \mathrm{~mm}$ & $0.21 \mathrm{~mm}$ \\
\hline 9 & 11 & 23 & $0.36 \mathrm{~mm}$ & $0.53 \mathrm{~mm}$ & $0.24 \mathrm{~mm}$ & $0.21 \mathrm{~mm}$ & $0.24 \mathrm{~mm}$ & $0.19 \mathrm{~mm}$ \\
\hline 9 & 11 & 31 & $0.52 \mathrm{~mm}$ & & $0.40 \mathrm{~mm}$ & $0.36 \mathrm{~mm}$ & & \\
\hline 10 & 8 & 23 & $0.17 \mathrm{~mm}$ & & $0.21 \mathrm{~mm}$ & & $0.28 \mathrm{~mm}$ & \\
\hline 10 & 8 & 24 & $0.25 \mathrm{~mm}$ & $0.38 \mathrm{~mm}$ & $0.24 \mathrm{~mm}$ & $0.37 \mathrm{~mm}$ & & \\
\hline 10 & 8 & 44 & $0.20 \mathrm{~mm}$ & $0.20 \mathrm{~mm}$ & $0.28 \mathrm{~mm}$ & $0.30 \mathrm{~mm}$ & $0.52 \mathrm{~mm}$ & $0.33 \mathrm{~mm}$ \\
\hline 11 & 24 & 16 & $0.53 \mathrm{~mm}$ & $0.39 \mathrm{~mm}$ & $0.52 \mathrm{~mm}$ & $0.22 \mathrm{~mm}$ & $0.21 \mathrm{~mm}$ & $0.18 \mathrm{~mm}$ \\
\hline 11 & 24 & 15 & & $0.11 \mathrm{~mm}$ & $0.29 \mathrm{~mm}$ & & $0.23 \mathrm{~mm}$ & $0.38 \mathrm{~mm}$ \\
\hline 11 & 24 & 13 & $0.21 \mathrm{~mm}$ & & $0.21 \mathrm{~mm}$ & $0.13 \mathrm{~mm}$ & $0.21 \mathrm{~mm}$ & $0.27 \mathrm{~mm}$ \\
\hline 11 & 24 & 27 & $0.22 \mathrm{~mm}$ & $0.19 \mathrm{~mm}$ & $0.17 \mathrm{~mm}$ & $0.38 \mathrm{~mm}$ & $0.29 \mathrm{~mm}$ & $0.25 \mathrm{~mm}$ \\
\hline 11 & 24 & 36 & $0.53 \mathrm{~mm}$ & $0.72 \mathrm{~mm}$ & $0.30 \mathrm{~mm}$ & $0.24 \mathrm{~mm}$ & $0.24 \mathrm{~mm}$ & $0.41 \mathrm{~mm}$ \\
\hline 11 & 24 & 35 & $0.32 \mathrm{~mm}$ & $0.27 \mathrm{~mm}$ & $0.23 \mathrm{~mm}$ & $0.28 \mathrm{~mm}$ & $0.38 \mathrm{~mm}$ & $0.33 \mathrm{~mm}$ \\
\hline 11 & 24 & 34 & $0.26 \mathrm{~mm}$ & $0.42 \mathrm{~mm}$ & $0.36 \mathrm{~mm}$ & $0.20 \mathrm{~mm}$ & $0.52 \mathrm{~mm}$ & \\
\hline 11 & 24 & 33 & $0.26 \mathrm{~mm}$ & & $0.31 \mathrm{~mm}$ & $0.17 \mathrm{~mm}$ & $0.40 \mathrm{~mm}$ & \\
\hline 11 & 24 & 43 & $0.32 \mathrm{~mm}$ & $0.34 \mathrm{~mm}$ & $0.17 \mathrm{~mm}$ & $0.35 \mathrm{~mm}$ & $0.15 \mathrm{~mm}$ & $0.26 \mathrm{~mm}$ \\
\hline 11 & 24 & 45 & $0.16 \mathrm{~mm}$ & $0.26 \mathrm{~mm}$ & $0.21 \mathrm{~mm}$ & $0.14 \mathrm{~mm}$ & & \\
\hline 11 & 24 & 46 & $0.32 \mathrm{~mm}$ & $0.45 \mathrm{~mm}$ & $0.35 \mathrm{~mm}$ & & & \\
\hline 12 & 26 & 23 & $0.27 \mathrm{~mm}$ & $0.28 \mathrm{~mm}$ & $0.58 \mathrm{~mm}$ & $0.18 \mathrm{~mm}$ & $0.35 \mathrm{~mm}$ & $0.16 \mathrm{~mm}$ \\
\hline 13 & 4 & 33 & $0.16 \mathrm{~mm}$ & $0.42 \mathrm{~mm}$ & $0.10 \mathrm{~mm}$ & $0.35 \mathrm{~mm}$ & & $0.26 \mathrm{~mm}$ \\
\hline 14 & 15 & 17 & $0.16 \mathrm{~mm}$ & $0.59 \mathrm{~mm}$ & $0.27 \mathrm{~mm}$ & $0.23 \mathrm{~mm}$ & $0.70 \mathrm{~mm}$ & $0.19 \mathrm{~mm}$ \\
\hline 14 & 15 & 11 & $0.46 \mathrm{~mm}$ & & $0.40 \mathrm{~mm}$ & & $0.18 \mathrm{~mm}$ & \\
\hline 14 & 15 & 21 & $0.47 \mathrm{~mm}$ & $0.43 \mathrm{~mm}$ & $0.24 \mathrm{~mm}$ & $0.18 \mathrm{~mm}$ & $0.21 \mathrm{~mm}$ & $0.19 \mathrm{~mm}$ \\
\hline 14 & 15 & 23 & $0.47 \mathrm{~mm}$ & $0.19 \mathrm{~mm}$ & $0.19 \mathrm{~mm}$ & & & \\
\hline 14 & 15 & 36 & $0.68 \mathrm{~mm}$ & $0.75 \mathrm{~mm}$ & $0.91 \mathrm{~mm}$ & $0.26 \mathrm{~mm}$ & $0.92 \mathrm{~mm}$ & \\
\hline 15 & 25 & 16 & & $0.82 \mathrm{~mm}$ & $0.77 \mathrm{~mm}$ & $0.30 \mathrm{~mm}$ & & $0.35 \mathrm{~mm}$ \\
\hline 15 & 25 & 15 & & $0.36 \mathrm{~mm}$ & & $0.40 \mathrm{~mm}$ & & \\
\hline 15 & 25 & 13 & $0.32 \mathrm{~mm}$ & $0.51 \mathrm{~mm}$ & $0.32 \mathrm{~mm}$ & $0.28 \mathrm{~mm}$ & $0.31 \mathrm{~mm}$ & $0.49 \mathrm{~mm}$ \\
\hline 15 & 25 & 11 & $0.40 \mathrm{~mm}$ & $0.39 \mathrm{~mm}$ & $0.24 \mathrm{~mm}$ & & & \\
\hline 15 & 25 & 21 & $0.51 \mathrm{~mm}$ & $0.39 \mathrm{~mm}$ & & $0.34 \mathrm{~mm}$ & & \\
\hline 15 & 25 & 22 & $0.31 \mathrm{~mm}$ & $0.44 \mathrm{~mm}$ & $0.32 \mathrm{~mm}$ & $0.22 \mathrm{~mm}$ & & \\
\hline 15 & 25 & 23 & $0.27 \mathrm{~mm}$ & $0.52 \mathrm{~mm}$ & $0.27 \mathrm{~mm}$ & $0.16 \mathrm{~mm}$ & $0.60 \mathrm{~mm}$ & $0.27 \mathrm{~mm}$ \\
\hline 15 & 25 & 35 & $0.40 \mathrm{~mm}$ & & $0.23 \mathrm{~mm}$ & & $0.26 \mathrm{~mm}$ & \\
\hline 15 & 25 & 33 & $0.33 \mathrm{~mm}$ & $0.49 \mathrm{~mm}$ & $0.28 \mathrm{~mm}$ & $0.22 \mathrm{~mm}$ & $0.42 \mathrm{~mm}$ & $0.36 \mathrm{~mm}$ \\
\hline 15 & 25 & 31 & $0.22 \mathrm{~mm}$ & $0.42 \mathrm{~mm}$ & & $0.23 \mathrm{~mm}$ & & $0.19 \mathrm{~mm}$ \\
\hline 15 & 25 & 45 & $0.60 \mathrm{~mm}$ & $0.36 \mathrm{~mm}$ & $0.18 \mathrm{~mm}$ & $0.68 \mathrm{~mm}$ & $0.32 \mathrm{~mm}$ & \\
\hline 15 & 25 & 47 & $0.32 \mathrm{~mm}$ & $0.35 \mathrm{~mm}$ & $0.32 \mathrm{~mm}$ & $0.22 \mathrm{~mm}$ & $0.25 \mathrm{~mm}$ & $0.53 \mathrm{~mm}$ \\
\hline 16 & 6 & 23 & $0.90 \mathrm{~mm}$ & $0.34 \mathrm{~mm}$ & $0.32 \mathrm{~mm}$ & $0.34 \mathrm{~mm}$ & $0.48 \mathrm{~mm}$ & $0.23 \mathrm{~mm}$ \\
\hline 16 & 6 & 33 & $0.31 \mathrm{~mm}$ & $0.25 \mathrm{~mm}$ & $0.56 \mathrm{~mm}$ & $0.53 \mathrm{~mm}$ & $0.26 \mathrm{~mm}$ & $0.18 \mathrm{~mm}$ \\
\hline 16 & 6 & 34 & $0.51 \mathrm{~mm}$ & $0.35 \mathrm{~mm}$ & $0.29 \mathrm{~mm}$ & $0.31 \mathrm{~mm}$ & & $0.36 \mathrm{~mm}$ \\
\hline
\end{tabular}


Table III. Data of 16 patients without antiresorptive drug medication (Group B).

\begin{tabular}{|c|c|c|c|c|c|c|c|c|}
\hline Patient No. & Number of teeth & Measured tooth & A & $\mathrm{B}$ & $\mathrm{C}$ & $\mathrm{D}$ & $\mathrm{E}$ & $\mathrm{F}$ \\
\hline 1 & 27 & 17 & $0.37 \mathrm{~mm}$ & $0.49 \mathrm{~mm}$ & $0.33 \mathrm{~mm}$ & $0.43 \mathrm{~mm}$ & $0.31 \mathrm{~mm}$ & \\
\hline 1 & 27 & 13 & $0.26 \mathrm{~mm}$ & & $0.16 \mathrm{~mm}$ & $0.20 \mathrm{~mm}$ & $0.37 \mathrm{~mm}$ & \\
\hline 1 & 27 & 12 & $0.56 \mathrm{~mm}$ & $0.27 \mathrm{~mm}$ & & $0.16 \mathrm{~mm}$ & $0.31 \mathrm{~mm}$ & $0.37 \mathrm{~mm}$ \\
\hline 1 & 27 & 27 & $0.58 \mathrm{~mm}$ & & $0.49 \mathrm{~mm}$ & & $0.31 \mathrm{~mm}$ & \\
\hline 1 & 27 & 36 & $0.31 \mathrm{~mm}$ & $0.11 \mathrm{~mm}$ & $0.20 \mathrm{~mm}$ & $0.27 \mathrm{~mm}$ & $0.22 \mathrm{~mm}$ & $0.20 \mathrm{~mm}$ \\
\hline 1 & 27 & 41 & $0.37 \mathrm{~mm}$ & $0.26 \mathrm{~mm}$ & $0.28 \mathrm{~mm}$ & $0.28 \mathrm{~mm}$ & & \\
\hline 1 & 27 & 42 & $0.20 \mathrm{~mm}$ & $0.37 \mathrm{~mm}$ & $0.28 \mathrm{~mm}$ & $0.28 \mathrm{~mm}$ & & \\
\hline 1 & 27 & 43 & $0.53 \mathrm{~mm}$ & $0.41 \mathrm{~mm}$ & $0.32 \mathrm{~mm}$ & $0.33 \mathrm{~mm}$ & $0.28 \mathrm{~mm}$ & $0.28 \mathrm{~mm}$ \\
\hline 1 & 27 & 45 & $0.41 \mathrm{~mm}$ & $0.30 \mathrm{~mm}$ & & $0.24 \mathrm{~mm}$ & $0.33 \mathrm{~mm}$ & $0.29 \mathrm{~mm}$ \\
\hline 2 & 18 & 27 & & & & $0.89 \mathrm{~mm}$ & & $0.27 \mathrm{~mm}$ \\
\hline 2 & 18 & 36 & $0.23 \mathrm{~mm}$ & $0.68 \mathrm{~mm}$ & $0.20 \mathrm{~mm}$ & $0.44 \mathrm{~mm}$ & & \\
\hline 2 & 18 & 35 & $0.34 \mathrm{~mm}$ & $0.37 \mathrm{~mm}$ & & $0.20 \mathrm{~mm}$ & & \\
\hline 3 & 19 & 13 & $0.47 \mathrm{~mm}$ & & $0.23 \mathrm{~mm}$ & & & \\
\hline 3 & 19 & 23 & $0.40 \mathrm{~mm}$ & & $0.45 \mathrm{~mm}$ & & & \\
\hline 3 & 19 & 25 & $0.64 \mathrm{~mm}$ & & $0.19 \mathrm{~mm}$ & & $0.32 \mathrm{~mm}$ & \\
\hline 3 & 19 & 45 & & $0.27 \mathrm{~mm}$ & & $0.12 \mathrm{~mm}$ & & \\
\hline 4 & 21 & 16 & $0.46 \mathrm{~mm}$ & $0.21 \mathrm{~mm}$ & $0.18 \mathrm{~mm}$ & $0.60 \mathrm{~mm}$ & $0.29 \mathrm{~mm}$ & \\
\hline 4 & 21 & 15 & $0.51 \mathrm{~mm}$ & $0.31 \mathrm{~mm}$ & $0.23 \mathrm{~mm}$ & $0.28 \mathrm{~mm}$ & $0.38 \mathrm{~mm}$ & $0.33 \mathrm{~mm}$ \\
\hline 4 & 21 & 13 & & & $0.39 \mathrm{~mm}$ & & $0.23 \mathrm{~mm}$ & $0.38 \mathrm{~mm}$ \\
\hline 4 & 21 & 12 & $0.41 \mathrm{~mm}$ & $0.45 \mathrm{~mm}$ & $0.28 \mathrm{~mm}$ & $0.19 \mathrm{~mm}$ & $0.43 \mathrm{~mm}$ & $0.43 \mathrm{~mm}$ \\
\hline 4 & 21 & 37 & $0.74 \mathrm{~mm}$ & $0.73 \mathrm{~mm}$ & & $0.38 \mathrm{~mm}$ & & \\
\hline 4 & 21 & 45 & $0.26 \mathrm{~mm}$ & $0.26 \mathrm{~mm}$ & $0.22 \mathrm{~mm}$ & $0.22 \mathrm{~mm}$ & & \\
\hline 5 & 5 & 37 & $0.95 \mathrm{~mm}$ & $0.55 \mathrm{~mm}$ & $0.46 \mathrm{~mm}$ & $0.43 \mathrm{~mm}$ & $0.28 \mathrm{~mm}$ & $0.23 \mathrm{~mm}$ \\
\hline 5 & 5 & 34 & $0.46 \mathrm{~mm}$ & $0.29 \mathrm{~mm}$ & & $0.58 \mathrm{~mm}$ & & $0.41 \mathrm{~mm}$ \\
\hline 5 & 5 & 43 & & $0.42 \mathrm{~mm}$ & & $0.65 \mathrm{~mm}$ & & $0.45 \mathrm{~mm}$ \\
\hline 6 & 12 & 13 & $0.38 \mathrm{~mm}$ & $0.31 \mathrm{~mm}$ & $0.21 \mathrm{~mm}$ & $0.48 \mathrm{~mm}$ & $0.36 \mathrm{~mm}$ & \\
\hline 6 & 12 & 23 & $0.33 \mathrm{~mm}$ & $0.22 \mathrm{~mm}$ & $0.37 \mathrm{~mm}$ & $0.25 \mathrm{~mm}$ & & $0.37 \mathrm{~mm}$ \\
\hline 6 & 12 & 36 & & $0.51 \mathrm{~mm}$ & $0.33 \mathrm{~mm}$ & $0.27 \mathrm{~mm}$ & $0.63 \mathrm{~mm}$ & $0.46 \mathrm{~mm}$ \\
\hline 6 & 12 & 35 & $0.61 \mathrm{~mm}$ & $0.25 \mathrm{~mm}$ & & $0.27 \mathrm{~mm}$ & & \\
\hline 6 & 12 & 34 & $0.91 \mathrm{~mm}$ & $0.56 \mathrm{~mm}$ & & & & $0.29 \mathrm{~mm}$ \\
\hline 6 & 12 & 33 & $0.77 \mathrm{~mm}$ & $0.44 \mathrm{~mm}$ & & & $0.69 \mathrm{~mm}$ & \\
\hline 7 & 26 & 15 & & $0.30 \mathrm{~mm}$ & & $0.37 \mathrm{~mm}$ & & \\
\hline 7 & 26 & 14 & & & & $0.27 \mathrm{~mm}$ & & $0.15 \mathrm{~mm}$ \\
\hline 7 & 26 & 13 & & $0.66 \mathrm{~mm}$ & & & $0.26 \mathrm{~mm}$ & $0.67 \mathrm{~mm}$ \\
\hline 7 & 26 & 23 & & $0.63 \mathrm{~mm}$ & & $0.28 \mathrm{~mm}$ & & \\
\hline 7 & 26 & 36 & $1.58 \mathrm{~mm}$ & $0.40 \mathrm{~mm}$ & $0.41 \mathrm{~mm}$ & $0.34 \mathrm{~mm}$ & $0.27 \mathrm{~mm}$ & \\
\hline 7 & 26 & 35 & $0.73 \mathrm{~mm}$ & & & $0.35 \mathrm{~mm}$ & & $0.36 \mathrm{~mm}$ \\
\hline 7 & 26 & 43 & $0.98 \mathrm{~mm}$ & $0.58 \mathrm{~mm}$ & $0.68 \mathrm{~mm}$ & $0.93 \mathrm{~mm}$ & $0.59 \mathrm{~mm}$ & \\
\hline 7 & 26 & 46 & & $0.39 \mathrm{~mm}$ & & $0.20 \mathrm{~mm}$ & $0.28 \mathrm{~mm}$ & $0.39 \mathrm{~mm}$ \\
\hline 8 & 25 & 17 & $0.59 \mathrm{~mm}$ & $0.47 \mathrm{~mm}$ & $0.15 \mathrm{~mm}$ & $0.42 \mathrm{~mm}$ & & \\
\hline 8 & 25 & 11 & $0.41 \mathrm{~mm}$ & $0.32 \mathrm{~mm}$ & $0.35 \mathrm{~mm}$ & $0.36 \mathrm{~mm}$ & $0.29 \mathrm{~mm}$ & $0.11 \mathrm{~mm}$ \\
\hline 8 & 25 & 21 & $0.27 \mathrm{~mm}$ & $0.40 \mathrm{~mm}$ & $0.42 \mathrm{~mm}$ & $0.51 \mathrm{~mm}$ & $0.25 \mathrm{~mm}$ & $0.37 \mathrm{~mm}$ \\
\hline 8 & 25 & 22 & & $0.48 \mathrm{~mm}$ & & $0.30 \mathrm{~mm}$ & & $0.26 \mathrm{~mm}$ \\
\hline 8 & 25 & 23 & $0.48 \mathrm{~mm}$ & $0.33 \mathrm{~mm}$ & $0.26 \mathrm{~mm}$ & $0.38 \mathrm{~mm}$ & $0.30 \mathrm{~mm}$ & $0.22 \mathrm{~mm}$ \\
\hline 8 & 25 & 37 & $0.70 \mathrm{~mm}$ & $0.80 \mathrm{~mm}$ & $0.27 \mathrm{~mm}$ & $0.25 \mathrm{~mm}$ & $0.50 \mathrm{~mm}$ & $0.30 \mathrm{~mm}$ \\
\hline 8 & 25 & 36 & $0.56 \mathrm{~mm}$ & $0.54 \mathrm{~mm}$ & & $0.14 \mathrm{~mm}$ & & $0.29 \mathrm{~mm}$ \\
\hline 8 & 25 & 34 & $0.64 \mathrm{~mm}$ & $0.25 \mathrm{~mm}$ & $0.32 \mathrm{~mm}$ & $0.49 \mathrm{~mm}$ & $0.59 \mathrm{~mm}$ & $0.56 \mathrm{~mm}$ \\
\hline 8 & 25 & 43 & $0.41 \mathrm{~mm}$ & $0.45 \mathrm{~mm}$ & $0.27 \mathrm{~mm}$ & $0.37 \mathrm{~mm}$ & & $0.29 \mathrm{~mm}$ \\
\hline 8 & 25 & 44 & & $0.77 \mathrm{~mm}$ & $0.34 \mathrm{~mm}$ & $0.43 \mathrm{~mm}$ & & \\
\hline 8 & 25 & 45 & $0.58 \mathrm{~mm}$ & $0.57 \mathrm{~mm}$ & & $0.19 \mathrm{~mm}$ & $0.43 \mathrm{~mm}$ & $0.32 \mathrm{~mm}$ \\
\hline 8 & 25 & 46 & $0.62 \mathrm{~mm}$ & $0.52 \mathrm{~mm}$ & $0.51 \mathrm{~mm}$ & $0.32 \mathrm{~mm}$ & $0.28 \mathrm{~mm}$ & \\
\hline 8 & 25 & 47 & $0.39 \mathrm{~mm}$ & $0.63 \mathrm{~mm}$ & $0.44 \mathrm{~mm}$ & $0.37 \mathrm{~mm}$ & $0.41 \mathrm{~mm}$ & $0.29 \mathrm{~mm}$ \\
\hline 9 & 26 & 16 & $0.50 \mathrm{~mm}$ & & $0.18 \mathrm{~mm}$ & & $0.24 \mathrm{~mm}$ & \\
\hline 9 & 26 & 12 & & $0.36 \mathrm{~mm}$ & & $0.30 \mathrm{~mm}$ & & $0.33 \mathrm{~mm}$ \\
\hline 9 & 26 & 25 & & $0.33 \mathrm{~mm}$ & & $0.28 \mathrm{~mm}$ & & $0.12 \mathrm{~mm}$ \\
\hline 9 & 26 & 26 & $0.48 \mathrm{~mm}$ & & $0.19 \mathrm{~mm}$ & & $0.20 \mathrm{~mm}$ & \\
\hline 9 & 26 & 37 & $0.50 \mathrm{~mm}$ & $0.45 \mathrm{~mm}$ & & $0.15 \mathrm{~mm}$ & & $0.28 \mathrm{~mm}$ \\
\hline 10 & 27 & 27 & $0.34 \mathrm{~mm}$ & $0.41 \mathrm{~mm}$ & $0.37 \mathrm{~mm}$ & $0.17 \mathrm{~mm}$ & $0.34 \mathrm{~mm}$ & \\
\hline 11 & 17 & 37 & $0.37 \mathrm{~mm}$ & $0.23 \mathrm{~mm}$ & & $0.26 \mathrm{~mm}$ & & \\
\hline
\end{tabular}


Table III. Continued

\begin{tabular}{|c|c|c|c|c|c|c|c|c|}
\hline Patient No. & Number of teeth & Measured tooth & A & B & $\mathrm{C}$ & $\mathrm{D}$ & E & $\mathrm{F}$ \\
\hline 11 & 17 & 35 & & $0.19 \mathrm{~mm}$ & & $0.27 \mathrm{~mm}$ & & $0.23 \mathrm{~mm}$ \\
\hline 11 & 17 & 33 & $0.53 \mathrm{~mm}$ & $0.31 \mathrm{~mm}$ & & $0.24 \mathrm{~mm}$ & & \\
\hline 11 & 17 & 32 & $0.32 \mathrm{~mm}$ & $0.58 \mathrm{~mm}$ & $0.28 \mathrm{~mm}$ & $0.35 \mathrm{~mm}$ & $0.14 \mathrm{~mm}$ & \\
\hline 11 & 17 & 46 & $0.24 \mathrm{~mm}$ & & $0.36 \mathrm{~mm}$ & & $0.28 \mathrm{~mm}$ & \\
\hline 12 & 24 & 16 & $0.26 \mathrm{~mm}$ & $0.31 \mathrm{~mm}$ & $0.23 \mathrm{~mm}$ & & & \\
\hline 12 & 24 & 15 & $0.38 \mathrm{~mm}$ & $0.41 \mathrm{~mm}$ & $0.21 \mathrm{~mm}$ & $0.38 \mathrm{~mm}$ & & $0.30 \mathrm{~mm}$ \\
\hline 12 & 24 & 14 & $0.40 \mathrm{~mm}$ & $0.44 \mathrm{~mm}$ & $0.34 \mathrm{~mm}$ & & $0.34 \mathrm{~mm}$ & \\
\hline 12 & 24 & 13 & $0.35 \mathrm{~mm}$ & $0.36 \mathrm{~mm}$ & $0.28 \mathrm{~mm}$ & & $0.25 \mathrm{~mm}$ & $0.21 \mathrm{~mm}$ \\
\hline 12 & 24 & 25 & & $0.46 \mathrm{~mm}$ & & $0.38 \mathrm{~mm}$ & & $0.19 \mathrm{~mm}$ \\
\hline 12 & 24 & 26 & $0.67 \mathrm{~mm}$ & & $0.63 \mathrm{~mm}$ & & $0.47 \mathrm{~mm}$ & \\
\hline 12 & 24 & 37 & $0.29 \mathrm{~mm}$ & $0.45 \mathrm{~mm}$ & $0.39 \mathrm{~mm}$ & $0.33 \mathrm{~mm}$ & & $0.38 \mathrm{~mm}$ \\
\hline 12 & 24 & 35 & $0.59 \mathrm{~mm}$ & $0.38 \mathrm{~mm}$ & $0.22 \mathrm{~mm}$ & $0.32 \mathrm{~mm}$ & $0.29 \mathrm{~mm}$ & $0.26 \mathrm{~mm}$ \\
\hline 12 & 24 & 33 & $0.56 \mathrm{~mm}$ & $0.27 \mathrm{~mm}$ & $0.21 \mathrm{~mm}$ & $0.48 \mathrm{~mm}$ & & \\
\hline 12 & 24 & 43 & $0.38 \mathrm{~mm}$ & $0.40 \mathrm{~mm}$ & & $0.44 \mathrm{~mm}$ & $0.34 \mathrm{~mm}$ & \\
\hline 12 & 24 & 46 & $0.32 \mathrm{~mm}$ & $0.48 \mathrm{~mm}$ & $0.13 \mathrm{~mm}$ & $0.33 \mathrm{~mm}$ & $0.35 \mathrm{~mm}$ & \\
\hline 13 & 2 & 23 & $0.54 \mathrm{~mm}$ & $0.64 \mathrm{~mm}$ & $0.54 \mathrm{~mm}$ & $0.23 \mathrm{~mm}$ & $0.23 \mathrm{~mm}$ & $0.37 \mathrm{~mm}$ \\
\hline 13 & 2 & 33 & $1.02 \mathrm{~mm}$ & $0.76 \mathrm{~mm}$ & $0.24 \mathrm{~mm}$ & $0.71 \mathrm{~mm}$ & $0.56 \mathrm{~mm}$ & $0.38 \mathrm{~mm}$ \\
\hline 14 & 8 & 23 & $0.32 \mathrm{~mm}$ & $0.27 \mathrm{~mm}$ & $0.36 \mathrm{~mm}$ & $0.18 \mathrm{~mm}$ & $0.43 \mathrm{~mm}$ & $0.14 \mathrm{~mm}$ \\
\hline 14 & 8 & 34 & $0.28 \mathrm{~mm}$ & & $0.20 \mathrm{~mm}$ & & $0.18 \mathrm{~mm}$ & \\
\hline 14 & 8 & 33 & $0.40 \mathrm{~mm}$ & & & $0.30 \mathrm{~mm}$ & & $0.22 \mathrm{~mm}$ \\
\hline 14 & 8 & 44 & $0.26 \mathrm{~mm}$ & $0.36 \mathrm{~mm}$ & & $0.68 \mathrm{~mm}$ & & \\
\hline 15 & 24 & 17 & $0.24 \mathrm{~mm}$ & $0.60 \mathrm{~mm}$ & $0.19 \mathrm{~mm}$ & $0.25 \mathrm{~mm}$ & $0.17 \mathrm{~mm}$ & \\
\hline 15 & 24 & 13 & $0.26 \mathrm{~mm}$ & & $0.28 \mathrm{~mm}$ & & & $0.77 \mathrm{~mm}$ \\
\hline 15 & 24 & 27 & & $0.17 \mathrm{~mm}$ & & $0.26 \mathrm{~mm}$ & & $0.36 \mathrm{~mm}$ \\
\hline 15 & 24 & 37 & & $0.40 \mathrm{~mm}$ & & $0.13 \mathrm{~mm}$ & & $0.30 \mathrm{~mm}$ \\
\hline 15 & 24 & 42 & $0.21 \mathrm{~mm}$ & $0.44 \mathrm{~mm}$ & & $0.26 \mathrm{~mm}$ & $0.32 \mathrm{~mm}$ & \\
\hline 15 & 24 & 43 & $0.49 \mathrm{~mm}$ & $0.58 \mathrm{~mm}$ & $0.39 \mathrm{~mm}$ & $0.30 \mathrm{~mm}$ & & \\
\hline 15 & 24 & 44 & $0.30 \mathrm{~mm}$ & $0.25 \mathrm{~mm}$ & $0.25 \mathrm{~mm}$ & & $0.24 \mathrm{~mm}$ & \\
\hline 16 & 13 & 21 & & $0.22 \mathrm{~mm}$ & $0.21 \mathrm{~mm}$ & $0.17 \mathrm{~mm}$ & $0.42 \mathrm{~mm}$ & $0.22 \mathrm{~mm}$ \\
\hline 16 & 13 & 43 & $0.41 \mathrm{~mm}$ & $0.26 \mathrm{~mm}$ & $0.19 \mathrm{~mm}$ & & & \\
\hline 16 & 13 & 42 & $0.23 \mathrm{~mm}$ & & $0.19 \mathrm{~mm}$ & & $0.41 \mathrm{~mm}$ & \\
\hline
\end{tabular}

patients $(25 \%)$ received oral bisphosphonates. Nine patients received zoledronate $(56.25 \%), 4$ patients alendronate $(25 \%)$, 2 patients ibandronate $(12.5 \%)$, and one patient received Dmab (6.25\%). Four of these patients received combined corticosteroid therapy (25\%) (Table I).

Analysis of the panoramic radiographs showed a mean width of the periodontal space of $0.43 \mathrm{~mm}$ in Group A with a confidence interval of $0.35-0.50 \mathrm{~mm}$ (Figures 1 and 2). Group B had a mean width of $0.37 \mathrm{~mm}$ with a confidence interval from $0.30 \mathrm{~mm}-0.44 \mathrm{~mm}$ (Figures 3 and 4).

In conclusion, Group A showed a measurable higher widening in the periodontal space of $0.06 \mathrm{~mm}(0.43 \mathrm{~mm} v \mathrm{~s}$. $0.37 \mathrm{~mm}$ ) in the mean (confidence interval of $0.05-0.17 \mathrm{~mm}$ ) (Table II), compared to the control Group B (Table III). The difference between the two groups was not statistically significant ( $p$-value 0.293).

\section{Discussion}

The aim of this study was to determine a possible correlation between the widening of the periodontal space and the occurrence of ARONJ.
However, a significant correlation between the widening of the periodontal space and ARONJ, as measured on panoramic radiographs, was not demonstrated. Although there is a slight widening of the periodontal space (mean difference between groups A and B of $0.06 \mathrm{~mm}$ ), this does not represent a clinically relevant difference or a clinically relevant effect of antiosteoclastic drug.

A preclinical study by Okamoto et al. analyzed the changes in the development of teeth as well as the width of the periodontal space in rats as an effect of BP treatment (33). They analyzed 40 rats for a total of 9 weeks, dividing them into 4 subgroups. Three of them were given different, and increasing doses of zoledronate, with the fourth group serving as a control. Zoledronate was applied over a period of 3 weeks. The first group was sacrificed one week after the last injection and the other ones were sacrificed 9 weeks after the last injection. In summary, there was a correlation between the narrowing of the PDS and increasing doses of BPs (33).

Another preclinical study by Sonis et al. reported certain osseous changes that occurred after tooth extractions in rats under BP treatment (34). The control group of rats did not 
show any significant changes, especially not in the trabecular bone parts, as well as no signs of radiographic or radiolucent lesions (34).

Although these preclinical models suggest that medication with BPs can induce changes in the PSW and adjacent osseous structures (34), similar changes were not detected in the patients participated in our study. Our results are in support of similar findings by the group of Koth et al., who recently evaluated clinical, radiographic and hematological findings in patients with antiresorptive drug therapy (35).

However, the measurement of the periodontal space in patients using panoramic radiographs might depend on the investigator. However, all possible measures were taken to exclude this kind of bias, since investigators were blinded for the subgroups.

In the study of Takaishi 2010 the authors managed to establish a reference, as they chose one healthy person to determine the grey shades used as a parameter to discriminate bone from tooth, while adjusting for example the brightness (36). Furthermore, the authors used aluminum step wedges pasted in panoramic films and received therefore a more reliable outcome (36).

Although the distortion is very high in panoramic radiographs, this examination provides a detailed overview of dental structures (37). For a single object securely positioned in the focal trough and perpendicular to the central X-ray beam, measurements may be trustworthy on clinical scale. However, once sterical relationships to other structures are evaluated, reliability must be questioned (38). One of the main functions panoramic radiographs is to give an overall impression, and if further investigation or a more detailed examination is required, usually a 3D imaging examination is performed $(13,38)$.

Additionally, panoramic radiographs often did not allow for an evaluation of all teeth and every part of the periodontal space, since parts of the image might suffer from overlaying structures or are blurred due to technical reasons. Therefore, evaluation of the periodontal space was restricted to those teeth where at least three measurements could be obtained.

In conclusion, measuring of the PDS using panoramic radiographs is still prone to investigator bias and technical restrictions. Similar difficulties were reported before, e.g. by the group of Arce in 2009 (31).

Overall, a clear correlation between ARONJ and the dental status has been established (39). In the study by Carmagnola et al., twice as much tooth extractions were found in patients with ARONJ after zoledronate therapy compared to healthy patients (39). However, our study as well as previous studies could not highlight any significant changes in PDS after antiresorptive drug treatment. Therefore, other parameters predictive of ARONJ still remain to be determined.

\section{Conclusion}

ARONJ is a multifactorial disease, with an increasing number of patients affected or at risk. Further research and analysis is necessary to better understand factors contributing to the occurrence of this disease. Especially panoramic radiographs, which are routinely carried out in these patients for an initial overview and evaluation of potential disease patterns, might be too inaccurate and uncertain to reliably detect affected areas, especially at early stages - and especially related to changes of the periodontal space and width.

There is a significant distortion of panoramic radiographs and potentially incoherent image quality, so panoramic radiographs might not be the best option for the particular evaluation of ARONJ. Although a very extensive and sophisticated interindividual comparison was performed to evaluate also slight changes of the periodontal space in patients with antiresorptive drug therapy, our results could not demonstrate changes in the PDS width, as demonstrated in preclinical models before. This is in line with our clinical experience, that panoramic radiographs are not sufficient to allow for a reliable assessment of stages and disease progression in ARONJ patients. Therefore, other radiographic examinations, such as cone beam computed tomography, magnetic resonance imaging or scintigraphies should be recommended for a safer evaluation of disease patterns in ARONJ patients.

\section{Conflicts of Interest}

The Authors declare, that there is no conflict of interest regarding this study.

\section{References}

1 Marx RE: Pamidronate (Aredia) and zoledronate (Zometa) induced avascular necrosis of the jaws: a growing epidemic. J Oral Maxillofac Surg 61(9): 1115-1117, 2003.

2 Koch FP, Yekta SS, Merkel C, Ziebart T and Smeets R: The impact of bisphosphonates on the osteoblast proliferation and Collagen gene expression in vitro. Head Face Med 6(1): 12-18, 2010.

3 Otto S, Abu-Id MH, Fedele S, Warnke PH, Becker ST, Kolk A, Mücke T, Mast G, Köhnke R, Volkmer E, Haasters F, Lieger O, Iizuka T, Porter S, Campisi G, Colella G, Ploder O, Neff A, Wiltfang J, Ehrenfeld M, Kreusch T, Wolff KD, Stürzenbaum SR, Schieker M and Pautke C: Osteoporosis and bisphosphonatesrelated osteonecrosis of the jaw: Not just a sporadic coincidence - A multi-centre study. J Craniomaxillofacial Surg 39(4): 272277, 2011.

4 Badros A, Weikel D, Salama A, Goloubeva O, Schneider A, Rapoport A, Fenton R, Gahres N, Sausville E, Ord R and Meiller $\mathrm{T}$ : Osteonecrosis of the jaw in multiple myeloma patients: clinical features and risk factors. J Clin Oncol 24(6): 945-952, 2006. 
5 Ruggiero SL, Dodson TB, Fantasia J, Goodday R, Aghaloo T, Mehrotra B and O'Ryan F: American Association of Oral and Maxillofacial Surgeons Position Paper on Medication-Related Osteonecrosis of the Jaw - 2014 update. J Oral Maxillofac Surg 72(10): 1938-1956, 2014.

6 Migliorati CA, Schubert MM, Peterson DE and Seneda LM: Bisphosphonate-associated osteonecrosis of mandibular and maxillary bone. Cancer 104(1): 83-93, 2005.

7 Murad O, Arora S, Farag A and Guber H: Bisphosphonates and Osteonecrosis of the Jaw: A Retrospective Study. Endocrine Practice 13(3): 232-238, 2007.

8 Saad F, Brown JE, Van Poznak C, Ibrahim T, Stemmer SM, Stopeck AT, Diel IJ, Takahashi S, Shore N, Henry DH, Barrios CH, Facon T, Senecal F, Fizazi K, Zhou L, Daniels A, Carrière $\mathrm{P}$ and Dansey R: Incidence, risk factors, and outcomes of osteonecrosis of the jaw: integrated analysis from three blinded active-controlled phase III trials in cancer patients with bone metastases. Ann Oncol 23(5): 1341-1347, 2012.

9 Bertoldo F, Santini D and Lo Cascio V: Bisphosphonates and osteomyelitis of the jaw: a pathogenic puzzle. Nat Clin Prac Oncol 4(12): 711-721, 2007.

10 Assaf AT, Zrnc TA, Riecke B, Wikner J, Zustin J, Friedrich RE, Heiland M, Smeets R and Gröbe A: Intraoperative efficiency of fluorescence imaging by Visually Enhanced Lesion Scope (VELscope ${ }^{\circledR}$ ) in patients with bisphosphonate related osteonecrosis of the jaw (BRONJ). J Craniomaxillofac Surg 42(5): 157-164, 2013.

11 Grötz K, Piesold JU, Al-Nawas B. Bisphosphonat-assoziierte Kiefernekrose (BP-ONJ) und andere Medikamenten-assoziierte Kiefernekrosen. AWMF Online, Register-N, 2012.

12 Amadori D, Aglietta M, Alessi B, Gianni L, Ibrahim T, Farina G, Gaion F, Bertoldo F, Santini D, Rondena R, Bogani P and Ripamonti CI: Efficacy and safety of 12-weekly versus 4-weekly zoledronic acid for prolonged treatment of patients with bone metastases from breast cancer (ZOOM): a phase 3, open-label, randomised, noninferiority trial. Lancet Oncol 14(7): 663-670, 2013.

13 Khan AA, Rios LP, Sándor GKB, Khan N, Peters E, Rahman MO, Clockie CM, Dore E and Dubois S: Bisphosphonate-associated osteonecrosis of the Jaw in Ontario: a survey of oral and maxillofacial surgeons. J Rheumatol 38(7): 1396-1402, 2011.

14 Scoletta M, Arduino PG, Pol R, Arata V, Silvestri S, Chiecchio $\mathrm{A}$ and Mozzati M: Initial experience on the outcome of teeth extractions in intravenous bisphosphonate-treated patients: A cautionary report. J Oral Maxillofac Surg 69(2): 456-462, 2011.

15 Stopeck AT, Lipton A, Body JJ, Steger GG, Tonkin K, de Boer RH, Lichinitser M, Fujiwara Y, Yardley DA, Viniegra M, Fan M, Jiang Q, Dansey R, Jun S and Braun A: Denosumab compared with zoledronic acid for the treatment of bone metastases in patients with advanced breast cancer: a randomized, doubleblind study. J Clin Oncol 28(35): 5132-5139, 2010

16 Tennis P, Rothman KJ, Bohn RL, Tan H, Zavras A, Laskarides $\mathrm{C}$, Calingaert B and Anthony MS: Incidence of osteonecrosis of the jaw among users of bisphosphonates with selected cancers or osteoporosis. Pharmacoepidemiology Drug Saf 21(8): 810$817,2012$.

17 Fizazi K, Lipton A, Mariette X, Body JJ, Rahim Y, Gralow JR, Gao G, Wu L, Sohn W and Jun S: Randomized Phase II trial of denosumab in patients with bone metastases from prostate cancer, breast cancer, or other neoplasms after intravenous bisphosphonates. J Clin Oncol 27(10): 1564-1571, 2009.
18 Fizazi K, Carducci M, Smith M, Damião R, Brown J, Karsh L, Milecki P, Shore N, Rader M, Wang H, Jiang Q, Tadros S, Dansey R and Goessl C: Denosumab versus zoledronic acid for treatment of bone metastases in men with castration-resistant prostate cancer: a randomised, double-blind study. Lancet 377(9768): 813-822, 2011.

19 Henry DH, Costa L, Goldwasser F, Hirsh V, Hungria V, Prausova J, Scagliotti GV, Sleeboom H, Spencer A, Vadhan-Raj S, von Moos R, Willenbacher W, Woll PJ, Wang J, Jiang Q, Jun S, Dansey R and Yeh H: Randomized, Double-Blind Study of Denosumab Versus Zoledronic Acid in the Treatment of Bone Metastases in Patients With Advanced Cancer (Excluding Breast and Prostate Cancer) or Multiple Myeloma. J Clin Oncol 29(9): 1125-1132, 2011

20 Scagliotti GV, Hirsh V, Siena S, Henry DH, Woll PJ, Manegold C, Solal-Celigny P, Rodriguez G, Krzakowski M, Mehta ND, Lipton L, García-Sáenz JA, Pereira JR, Prabhash K, Ciuleanu TE, Kanarev V, Wang H, Balakumaran A and Jacobs I: Overall survival improvement in patients with lung cancer and bone metastases treated with denosumab versus zoledronic acid: subgroup analysis from a randomized phase 3 study. J Thorac Oncol 7(12): 1823-1829, 2012.

21 Smith MR, Egerdie B, Toriz NH, Feldman R, Tammela TLJ, Saad F, Heracek J, Szwedowski M, Ke C, Kupic A, Leder BZ and Goessl C; Denosumab HALT Prostate Cancer Study Group: Denosumab in men receiving androgen-deprivation therapy for prostate cancer. N Engl J Med 361(8): 745-755, 2009.

22 Smith MR, Saad F, Oudard S, Shore N, Fizazi K, Sieber P, Tombal B, Damiao R, Marx G, Miller K, Van Veldhuizen P, Morote J, Ye Z, Dansey R and Goessl C: Denosumab and bonemetastasis-free survival in men with castration-resistant prostate cancer: results of a phase 3, randomised, placebo-controlled trial. Lancet 379(9810): 39-46, 2011.

23 Khan AA, Morrison A, Hanley DA, Felsenberg D, McCauley LK, O'Ryan F, Reid IR, Ruggiero SL, Taguchi A, Tetradis S, Watts NB, Brandi ML, Peters E, Guise T, Eastell R, Cheung AM, Morin SN, Masri B, Cooper C, Morgan SL, ObermayerPietsch B, Langdahl BL, Al Dabagh R, Davison KS, Kendler DL, Sándor GK, Josse RG, Bhandari M, El Rabbany M, Pierroz DD, Sulimani R, Saunders DP, Brown JP and Compston J; International Task Force on Osteonecrosis of the Jaw. Diagnosis and management of osteonecrosis of the jaw: a systematic review and international consensus. J Bone Min Res 30(1): 323, 2015.

24 Assaf AT, Zrnc TA, Remus CC, Adam G, Zustin J, Heiland M, Friedrich RE and Derlin T: Intraindividual comparison of preoperative $99 \mathrm{mTc}-\mathrm{MDP}$ SPECT/CT and intraoperative and histopathological findings in patients with bisphosphonate- or denosumab-related osteonecrosis of the jaw. $\mathbf{J}$ Craniomaxillofacial Surg 43(8): 1461-1469, 2015.

25 Yoneda T, Hagino H, Sugimoto T, Ohta H, Takahashi S, Soen S, Taguchi A, Nagata T, Urade M, Shibahara T and Toyosawa S: Antiresorptive agent-related osteonecrosis of the jaw: Position Paper 2017 of the Japanese Allied Committee on Osteonecrosis of the Jaw. J Bone Miner Metab 35(1): 6-19, 2017.

26 Hasegawa Y, Kawabe M, Kimura H, Kurita K, Fukuta J and Urade M: Influence of dentures in the initial occurrence site on the prognosis of bisphosphonate-related osteonecrosis of the jaws: a retrospective study. Oral Surg Oral Med Oral Pathol Oral Radiol 114(3): 318-324, 2012. 
27 Vahtsevanos K, Kyrgidis A, Verrou E, Katodritou E, Triaridis S, Andreadis CG, Boukovinas I, Koloutsos GE, Teleioudis Z, Kitikidou K, Paraskevopoulos P, Zervas K and Antoniades K: Longitudinal cohort study of risk factors in cancer patients of bisphosphonate-related osteonecrosis of the jaw. J Clin Oncol 27(32): 5356-5362, 2009.

28 Durie BG, Katz M and Crowley J: Osteonecrosis of the Jaw and Bisphosphonates. N Engl J Med 353(1): 99-102, 2005.

29 Dimitrakopoulos I, Magopoulos C and Karakasis D: Bisphosphonate-induced avascular osteonecrosis of the jaws: a clinical report of 11 cases. Int J Oral Maxillofac Surg 35(7): 588593, 2017.

30 Poxleitner P, Engelhardt M, Schmelzeisen R and Voss P: The Prevention of Medication-related Osteonecrosis of the Jaw. Dtsch Arztebl Int 114(5): 63-69, 2017.

31 Arce K, Assael LA, Weissman JL and Markiewicz MR: Imaging Findings in Bisphosphonate-Related Osteonecrosis of Jaws. J Oral Maxillofac Surg 67(5): 75-84, 2009.

32 Fleisher KE, Welch G, Kottal S, Craig RG, Saxena D and Glickman RS. Predicting risk for bisphosphonate-related osteonecrosis of the jaws: CTX versus radiographic markers. Oral Surg, Oral Med, Oral Pathol, Oral Radiol and Endodont 110(4): 509-516, 2010.

33 Okamoto Y, Hirota M, Monden Y, Murata S, Koyama C, Mitsudo K, Iwai T, Ishikawa $\mathrm{Y}$ and Tohnai I: High-dose zoledronic acid narrows the periodontal space in rats. Int $\mathrm{J}$ Oral Maxillofac Surg 42(5): 627-631, 2012.

34 Sonis ST, Watkins BA, Lyng GD, Lerman MA and Anderson $\mathrm{KC}$ : Bony changes in the jaws of rats treated with zoledronic acid and dexamethasone before dental extractions mimic bisphosphonate-related osteonecrosis in cancer patients. Oral Oncol 45(2): 164-172, 2009.
35 Koth VS, Figueiredo MA, Salum FG and Cherubini K: Interrelationship of clinical, radiographic and haematological features in patients under bisphosphonate therapy. Dentomaxillofac Rad 46(4): 20160260, 2017.

36 Takaishi Y, Ikeo T, Nakajima M, Miki T and Fujita T: A pilot case-control study on the alveolar bone density measurement in risk assessment for bisphosphonate-related osteonecrosis of the jaw. Osteoporos Int 21(5): 815-825, 2010.

37 Bianchi SD, Scoletta M, Cassione FB, Migliaretti G and Mozzati $\mathrm{M}$ : Computerized tomographic findings in bisphosphonateassociated osteonecrosis of the jaw in patients with cancer. Oral Surg, Oral Med, Oral Pathol, Oral Radiol and Endodont 104(2): 249-258, 2007.

38 Riecke B, Friedrich RE, Schulze D, Loos C, Blessmann M, Heiland $\mathrm{M}$ and Wikner $\mathrm{J}$ : Impact of malpositioning on panoramic radiography in implant dentistry. Clin Oral Invest 19(4): 781-790, 2015.

39 Carmagnola D, Celestino S and Abati S: Dental and periodontal history of oncologic patients on parenteral bisphosphonates with or without osteonecrosis of the jaws: A pilot study. Oral Surg, Oral Med, Oral Pathol, Oral Radiol and Endodont 106(6): 1015,2008 .
Received July 27, 2018

Revised August 14, 2018

Accepted August 19, 2018 